\title{
Radiation, Charged Particles
}

National Cancer Institute

\section{Source}

National Cancer Institute. Radiation, Charged Particles. NCI Thesaurus. Code C18982.

A particle which carries a positive or negative electrical charge, typically an ionized atom or molecule, or an electron. 